\title{
El problema del drenaje en Tijuana y San Diego: una fuente de conflicto binacional entre México y Estados Unidos
}

\author{
Roberto Sánchez*
}

\begin{abstract}
En este trabajo se aborda el problema del drenaje en Tijuana; se expone ampliamente la situación actual, destacando los principales aspectos de la negociación binacional que se ha realizado; se establece un enfoque regional del problema con el objeto de deslindar la responsabilidad real de Tijuana y San Diego en la contaminación del litoral; se analizan los aspectos susceptibles de incidir en la selección de alternativas de solución, entre los que destaca la construcción de una planta binacional. Por último se estudian las posibles estrategias de negociación que podría adoptar México. Entre ellas se menciona la posibilidad de pagos en especie y la de crear alianzas con grupos estadunidenses que favorecen la cooperación entre los dos paises.
\end{abstract}

La importancia del medio ambiente en las relaciones binacionales entre México y Estados Unidos se ha incrementado de manera significativa desde la firma del convenio binacional en 1983. A partir de este acuerdo se empiezan a integrar temas que hasta ese momento se habían negociado de manera aislada. Se estructuran los mecanismos de negociación entre ambos países para la solución de estòs problemas, y se empiezan a dar los primeros pasos para llevar a cabo los acuerdos logrados.

Las negociaciones sobre problemas ambientales han tenido otros beneficios para la relación binacional México-Estados Unidos. El medio ambiente fue el único tema sobre el que se lograron acuerdos en las reuniones de presidentes de estos dos países entre 1983 y 1986. Esto permitió presentar una imagen de continuidad en la relación bilateral que ha ayudado a contrarrestar, en alguna medida, la tensión y el distanciamiento ocasionado por la falta de consenso en la negociación de los otros grandes temas de la relación: trabajadores indocumentados, narcotráfico, deuda externa y relaciones comerciales.

Sin embargo, la negociación binacional sobre problemas ambientales ha enfrentado periodos de fuertes tensiones. El problema del drenaje de Tijuana es un buen ejemplo, ya que después de años de negociación aún no logra una solución satisfactoria. La perspectiva actual presenta a México como una fuente contaminante que pone en peligro las playas, la industria turística y la salud pública de los habitantes de San Diego.

Ésta es la imagen que los medios masivos de comunicación han difundido en Estados Unidos y bajo la cual se inició la negociación binacio-

* Investigador del Departamento del Medio Ambiente y Estudios Urbanos de El Colegio de la Frontera Norte. 
nal. El efecto ha sido que el proceso de negociación del problema de Tijuana se ha caracterizado por una actitud demandante de la parte estadunidense exigiendo una solución unilateral. En este sentido, dos factores han sido determinantes para que México haya aceptado negociar en estos términos: 1) la falta de información sobre los problemas ambientales transfronterizos - en especial lo que concierne a las fuentes en Estados Unidos y al impacto real de las fuentes contaminantes mexicanas en ese país - ha impedido que la parte mexicana pueda demandar una definición completa de los problemas; 2) la asimetría de poder que caracteriza la estructura binacional de México con Estados Unidos (Ojeda, 1983) ha deteriorado la desigual capacidad de negociación de la parte mexicana (Sánchez, 1988).

El momento actual en la negociación binacional de este problema es especialmente importante debido al reciente incremento en las derramas del efluente proveniente de Tijuana a través de la frontera, pero sobre todo, porque las dos ciudades se encuentran a punto de iniciar inversiones considerables para ampliar su sistema de drenaje: Tijuana iniciará la construcción de una nueva planta en la parte este de la ciudad; San Diego restructurará completamente su sistema y ampliará su tratamiento de primario avanzado a secundario con una inversión superior a 1500 millones de dólares. Formando parte de estas inversiones se menciona en Estados Unidos la posibilidad de construir una planta binacional Tijuana-San Diego, como una de las mejores alternativas para una solución a largo plazo al problema de Tijuana. Ésta es la segunda vez que una propuesta de este tipo se presenta como solución al conflicto binacional. En la ocasión anterior, Estados Unidos se opuso a cualquier tipo de ayuda y cooperación con México para llevar a cabo esta obra.

En esta coyuntura México puede utilizar mejores argumentos de negociación para la nueva ronda de negociaciones binacionales. Un aspecto clave será superar la rígida actitud adoptada por Estados Unidos durante la primera ronda de negociaciones en 1984 y 1985 . Para este fin, es importante que México trate de cambiar la imagen que lo presenta como la fuente contaminante del litoral de San Diego, lo cual sería factible a través de una perspectiva regional del problema que incluya la responsabilidad de San Diego en esta contaminación. El estudio de otras posibles alternativas al problema de Tijuana, incluyendo la construcción de la planta binacional, puede resultar en beneficio de una solución más permanente.

Este trabajo pretende ser una contribución para solventar el problema del drenaje en Tijuana. La primera parte presenta los principales aspectos de la negociación binacional llevada a cabo hasta el momento. La intención es proporcionar al lector un marco de referencia amplio que explique la situación actual, en especial los aspectos que impidieron lograr una solución satisfactoria. La segunda parte establece un enfoque regional del problema al estudiar el drenaje en Tijuana y San Diego. El objeti- 
vo principal es definir la responsabilidad real de cada ciudad en la contaminación del litoral trasfronterizo. La última parte del trabajo considera las implicaciones para México de la situación actual, en especial los aspectos que pueden incidir en la selección de alternativas de solución al problema de Tijuana, y las estrategias de negociación que de ellos se pueden derivar.

\section{La negociación binacional}

Uno de los logros del acuerdo binacional de 1983 fue la firma del anexo número uno en junio de 1985. En este anexo ambas partes aceptan la propuesta de México para solucionar el problema de Tijuana. El programa propuesto por México cubre las necesidades de Tijuana hasta el año 2000 para una población estimada de 1300000 habitantes y una capacidad de tratamiento de las aguas servidas de $2.6 \mathrm{~m}^{3} / \mathrm{seg}$. El proyecto prevé ampliar el sistema de drenaje de Tijuana y dividirlo en dos subsistemas: un subsistema poniente y uno oriente, cada uno con una planta de tratamiento. Siguiendo este acuerdo, la Sedue construyó una nueva estación de bombeo número 1 en 1986; se continuó ampliando la red de drenaje de la ciudad, en especial en las zonas en donde se originan la mayor parte de las derramas trasfronterizas, y se terminó una planta de tratamiento primario avanzado en 1987 en San Antonio del Mar dentro del subsistema poniente.

Esta planta se compone de tres lagunas de oxidación con una capacidad de $1.1 \mathrm{~m}^{3} / \mathrm{seg}$ (aproximadamente la totalidad del drenaje recolectado en Tijuana hasta 1988) ${ }^{1}$ y tuvo un costo de 7000 millones de pesos. A principios de 1987 la planta inició su periodo de pruebas, siendo éstas suspendidas en octubre de ese mismo año al presentarse importantes filtraciones en dos de las lagunas. Aunque las obras de reparación terminaron en febrero de 1988, con un costo adicional de 2500 millones de pesos, la Sedue ha extendido unos meses más el periodo de pruebas de las lagunas.

El subsistema oriente se basa en la futura construcción de una planta de tratamiento en el arroyo del Alamar con una capacidad de $1.5 \mathrm{~m}^{3} / \mathrm{seg}$ hasta el año 2000. La Sedue programó empezar a operar la planta en 1988, pero en el mejor de los casos esto no sucederá hasta 1990 , debido a que su construcción aún no se inicia. El subsistema oriente dará servicio en la parte este de la ciudad a una población de casi 900000 habitantes.

La solución adoptada por México para el drenaje de Tijuana no es, qũizás, la mejor alternativá técnica posible. El sistema de transporte del drenaje hasta la planta de tratamiento en la costa en el sistema poniente

${ }^{1}$ Esta cantidad corresponde a sólo $60 \%$ de la población de Tijuana que actualmente cuenta con servicio de drenaje. 
implica riesgos y es costoso en su operación. ${ }^{2}$ En el subsector oriente el drenaje tratado será vertido al río Tijuana, lo cual afectaría el ecosistema del estuario del mismo nombre al otro lado de la frontera.

Es probable que México hubiera optado por combinar mejores àlternativas técnicas al problema si la situación, en ese momento, lo hubiera permitido. La actitud poco flexible de Estados Unidos durante la negociación, preocupándose de que México tratara su drenaje al sur de la frontera, bloqueó cualquier intento de cooperación. ${ }^{3}$ Esta actitud, junto con la presión de la prensa y las autoridades estadunidenses sobre la parte mexicana demandando una solución rápida al problema, provocó una reacción nacionalista en la parte mexicana que redujo aún más las alternativas de solución. México se vio obligado a presentar una rápida respuesta que impidió considerar otras posibles alternativas de solución, entre ellas la construcción de una planta binacional.

La negociación del problema de Tijuana durante 1984 y 1985 se caracterizó por una actitud que algunos autores han denominado el principio de "el que contamina paga" (Mumme y Nalven, 1986). ${ }^{4}$ Bajo este principio, la administración Reagan ha mostrado una nula consideración a la situación económica de México y su capacidad de inversión en la solución de problemas no prioritarios a nivel nacional en momentos de crisis. La Oficina de Administración y Presupuesto (Office of Management and Budget) y el Departamento de Estado de la administración Reagan se opusieron fuertemente a ofrecer ayuda financiera estadunidense a la parte mexicana para la solución de problemas ambientales trasfronterizos actuales, en especial en el caso del drenaje de Tijuana. Estas dependencias consideraron que cualquier ayuda financiera establecería un precedente inaceptable para la negociación de otros problemas ambientales trasfronterizos (Nalven, 1986). Se argumentó que la fuente del problema es mexicana, y por lo tanto es su responsabilidad solucionarlo sin ayuda finaciera de Estados Unidos. Para la administración Reagan, la preocupación principal de Estados Unidos era centrar su atención en lograr que México tratara sus aguas servidas en el sur de la frontera (Nalven, 1986).

Por su parte, México tampoco intentó presentar una visión detallada del problema debido básicamente a la poca información con que contaba

${ }^{2}$ El sistema es básicamente el mismo que existia en Tijuana con algunas mejoras en el equipo. Como antes, el drenaje debe ser elevado más de 100 metros para llegar a la costa.

${ }^{3}$ La única alternativa de cooperación presentada por Estados Unidos fue la construcción de una planta binacional para tratar efluentes de San Diego y Tijuana. No obstante, la propuesta dejaba fuera toda posibilidad real de una participación mexicana. México debía pagar $\mathbf{4 0 0}$ millones de dólares, de un total de $\mathbf{7 4 0}$ millones, por la construcción de la planta. Ante la negativa mexicana para participar en este proyecto, la actitud estadunidense se tornó más rígida en contra de cualquier posible cooperación.

"Esta actitud tiene efectos similares a lo que se conoce como "la doctrina Harmon" en el caso de los problemas del agua superficial (Carroll, 1986). 
en ese momento sobre las fuentes contaminantes en San Diego, y al impacto de las derramas del efluente de Tijuana en el otro lado de la frontera. La posición de México en la negociación binacional fue vulnerable y con pocos argumentos para su defensa. Esto facilitó la actitud demandante y poco flexible de Estados Unidos en la negociación llevada a cabo durante 1984 y 1985, dejando poco espacio para la cooperación y obligando a México a buscar una solución unilateral para controlar las derramas del drenaje de Tijuana.

Una situación similar a la que se presentó en 1984 y 1985 está por repetirse. México está obligado a controlar nuevas derramas de aguas servidas a través de la frontera, especialmente en la parte este de Tijuana. Una nueva ola de.presión hacia México empieza a sentirse. Fallas en la operación de la planta de tratamiento de San Antonio del Mar, unos cuantos meses después del inicio de sus operaciones, han dado lugar a nuevas críticas hacia México en San Diego (The San Diego Union, 6 de noviembre de 1987). Dichas críticas argumentaban de nuevo la incapacidad de los mexicanos para resolver el problema del drenaje de Tijuana. México ha sido objeto de este tipo de críticas durante todo el proceso de la negociación binacional. En cada oportunidad, diversas autoridades, grupos de San Diego y la prensa local, han expresado su desconfianza hacia México y sus dudas sobre su capacidad para operar el sistema de drenaje de Tijuana. Estos críticos no han tenido en cuenta que problemas similares suceden en San Diego. Por el contrario, su actitud es la de exagerar los problemas de Tijuana y la forma como éstos afectan a San Diego.

Uno de los argumentos favoritos es el compromiso asumido por el entonces secretario de la Sedue, Manuel Camacho, en una entrevista de prensa celebrada después de la ceremonia de inicio de operaciones de la planta de tratamiento en San Antonio del Mar en enero de 1987. Como respuesta a la pregunta de si México dejaría de contaminar las playas de San Diego con la operación de la nueva planta, Camacho se comprometió a asegurar que México dejaría de contaminar las playas de San Diego (The San Diego Union, 29 de enero de 1987). Este hecho es de suma importancia por tratarse de la palabra de un secretario de Estado, y por haberse realizado en el momento en que el gobierno presentó el resultado de su esfuerzo para resolver el problema. México no ha logrado controlar las derramas de aguas servidas a través de la frontera y la planta ha tenido problemas en su operación. Esto ha facilitado que la prensa de San Diego dude de la palabra de Camacho y de la convicción de México para solucionar el problema (véanse Los Angeles Times, 17 de febrero de 1987; The San Diego Union, 27 de octubre de 1987 y Los Angeles Times, 16 de noviembre de 1987), a pesar del esfuerzo realizado para construir la planta de tratamiento y mejorar el sistema actual.

El incidente refuerza la necesidad de una definición real de la responsabilidad de México en la contaminación del litoral. Si bien las derra- 
mas del efluente de Tijuana a través del río Tijuana contaminan las playas de Imperial Beach en el extremo sur de San Diego, en ningún momento son responsables de la contaminación del resto de las playas de esa ciudad, como se insinúa en la prensa estadunidense. Importantes playas de San Diego son efectivamente contaminadas por derramas de aguas servidas, pero éstas son debidas a fallas en el sistema de drenaje de esa ciudad, según se documenta en este trabajo.

\section{El drenaje de Tijuana}

Tijuana ha padecido problemas de drenaje a lo largo de su historia. En sus primeros años, las aguas servidas de la ciudad eran vertidas directamente al río Tijuana. En 1938, Tijuana y San Diego utilizaban un emisor submarino para la descarga de su efluente en el litoral. El emisor se encontraba frente a la linea internacional en la zona ahora conocida como "Border Field". Tijuana tenía entonces 16000 habitantes (Meyer, 1983, citado por Conway y Álvarez, 1987).

A partir de la década de los cincuenta, la población y la superficie urbana de Tijuana crecieron aceleradamente. La generación de efluente se expandió siguiendo el crecimiento de la ciudad. A principios de la década de los sesenta, Tijuana construyó su primer sistema para el transporte de drenaje separado del de San Diego. Este sistema recolectaba el efluente de la ciudad para transportarlo y descargarlo en la costa cerca de Rosarito. En su trayecto, el efluente era elevado más de 100 metros cerca de la linea internacional a través de dos estaciones de bombeo.

Este sistema de transporte del drenaje de Tijuana tuvo constantes problemas -ruptura de colectores y fallas en la operación de las bombasocasionados por una deficiente operación pero también por el rápido incremento en el efluente y la incapacidad de la ciudad para expandir su red de drenaje al mismo ritmo. Las derramas más graves sucedian en la antigua estación de bombeo número 1 , donde se concentraba el efluente de la ciudad para ser bombeado a la costa (Meyer, 1983, citado por Conway y Álvarez, 1987). Esto daba lugar a derramas del efluente a través de la frontera, que fueron objeto de constantes protestas por parte de San Diego.

En un intento por controlar estas derramas, el gobierno federal de México y la ciudad de San Diego acordaron en 1965 la construcción de una conexión de emergencia entre el sistema de drenaje de Tijuana y el de San Diego. El acuerdo permitía a Tijuana enviar parte de sus aguas servidas a la planta de Point Loma, en San Diego, para su tratamiento, en caso de fallas en la operación del sistema de Tijuana (Conway, Salgado y Álvarez, 1987). El uso de la conexión de emergencia se incrementó con los 
años, así como la imposibilidad del sistema de drenaje de Tijuana de controlar el constante incremento de su efluente.

A principios de la década de los ochenta, Tijuana usaba la capacidad total de la conexión de emergencia las 24 horas del día, es decir 13 millones de galones por día (equivalentes a aproximadamente la mitad del drenaje recolectado en Tijuana) (Waisman, 1984). San Diego decidió cancelar el acuerdo en 1986 argumentando un excesivo costo en el servicio (Tijuana seguía pagando la cuota pactada 20 años antes para el tratamiento de su drenaje). No obstante, la conexión entre los dos sistemas se mantiene actualmente para casos de emergencia. ${ }^{5}$

Las continuas derramas de las aguas servidas de Tijuana en diversos puntos de la frontera dieron lugar al conflicto binacional como lo conocemos ahora. Básicamente existen tres partes por donde el efluente cruza la frontera: 1) el rio Tijuana, 2) a lo largo de la avenida internacional entre Tijuana y playas de Tijuana, básicamente en la zona de los cañones que desembocan en la frontera y, 3) las descargas en el litoral en playas de Tijuana (véase la fig. 1).

En el caso de las derramas en el rio Tijuana, éstas se deben a la saturación o la ruptura de colectores en la parte este de la ciudad. Una parte considerable del efluente recolectado en esa zona, sobre todo en la Mesa de Otay, no logra llegar al colector central. El efluente, que fluye hacia el río Tijuana siguiendo el dren natural de la zona, cruza la frontera y desemboca en el estuario del río Tijuana y en la playa de Border Field (véase la fig. 1).

Una parte importante del conflicto se debió a que el gobierno de California declaró este estuario zona de protección natural a finales de 1982. Las protestas por el derrame de drenaje a través del río Tijuana se incrementaron a partir de la afectación y perturbación del estuario. ${ }^{6}$ Sin embargo, las tres cuartas partes de la cuenca del río Tijuana, de la cual depende el estuario, se encuentran en México. Es paradójico que las autoridades de San Diego y el gobierno de California no hayan invitado oficialmente a México a participar en la conservación del estuario, cuando las tres cuartas partes del río Tijuana se encuentran en territorio mexicano. La conservación ecológica del estuario es incuestionable, pero para esto es necesario establecer una cooperación entre las dos partes afectadas. Desgraciadamente hasta ahora ha sucedido lo contrario. El estuario ha sido usado como un factor de presión política contra México, y no se ha establecido ningun tipo de comunicación entre las agencias encargadas de su conservación y las autoridades mexicanas.

\footnotetext{
${ }^{5}$ En el transcurso de los dos últimos años esta conexión se ha usado sólo por unas cuantas horas (The San Diego Union, 15 de diciembre de 1986).

${ }^{6}$ Véase por ejemplo el artículo en el The Son Diego Union, 26 de septiembre de 1985).
} 


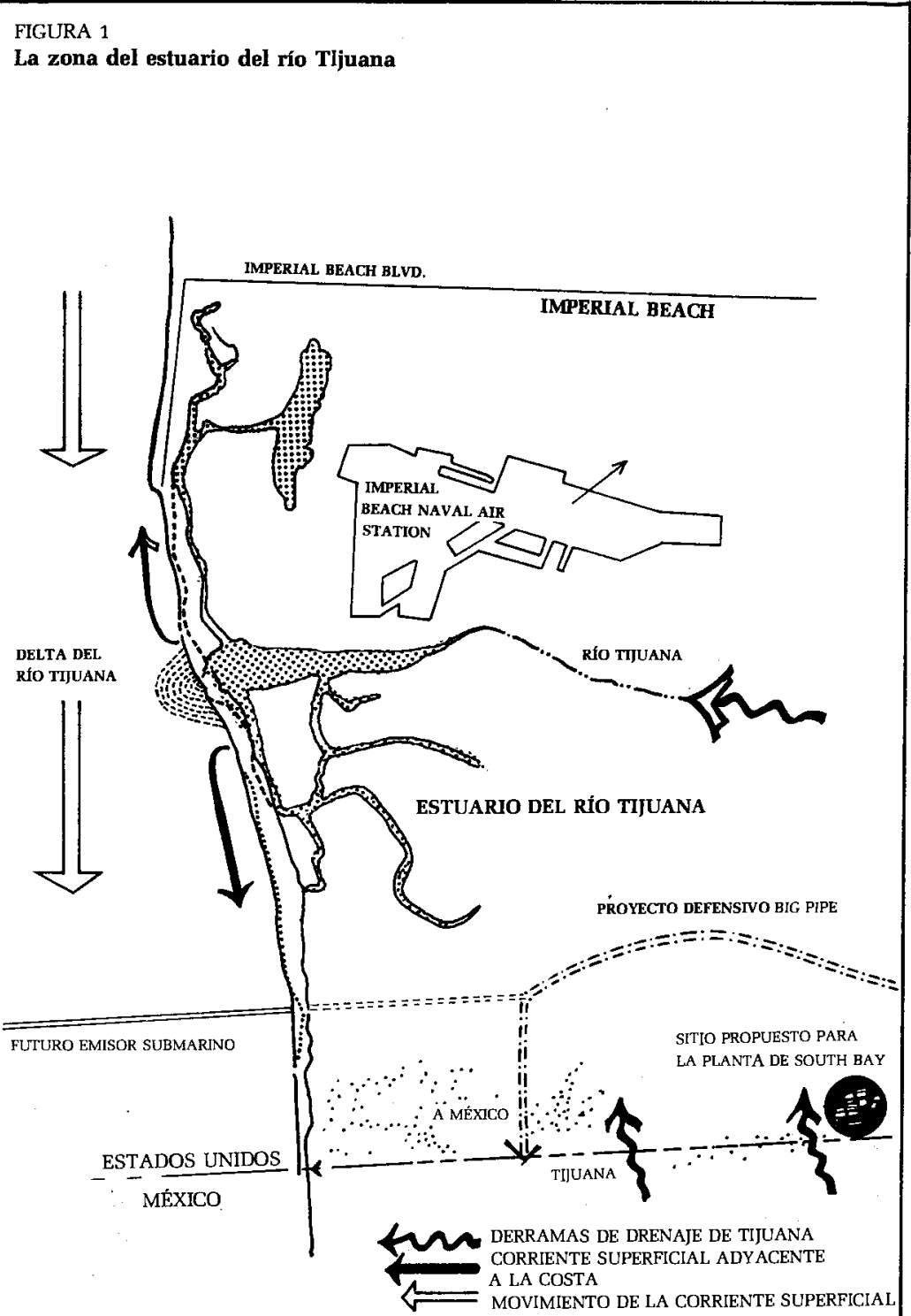




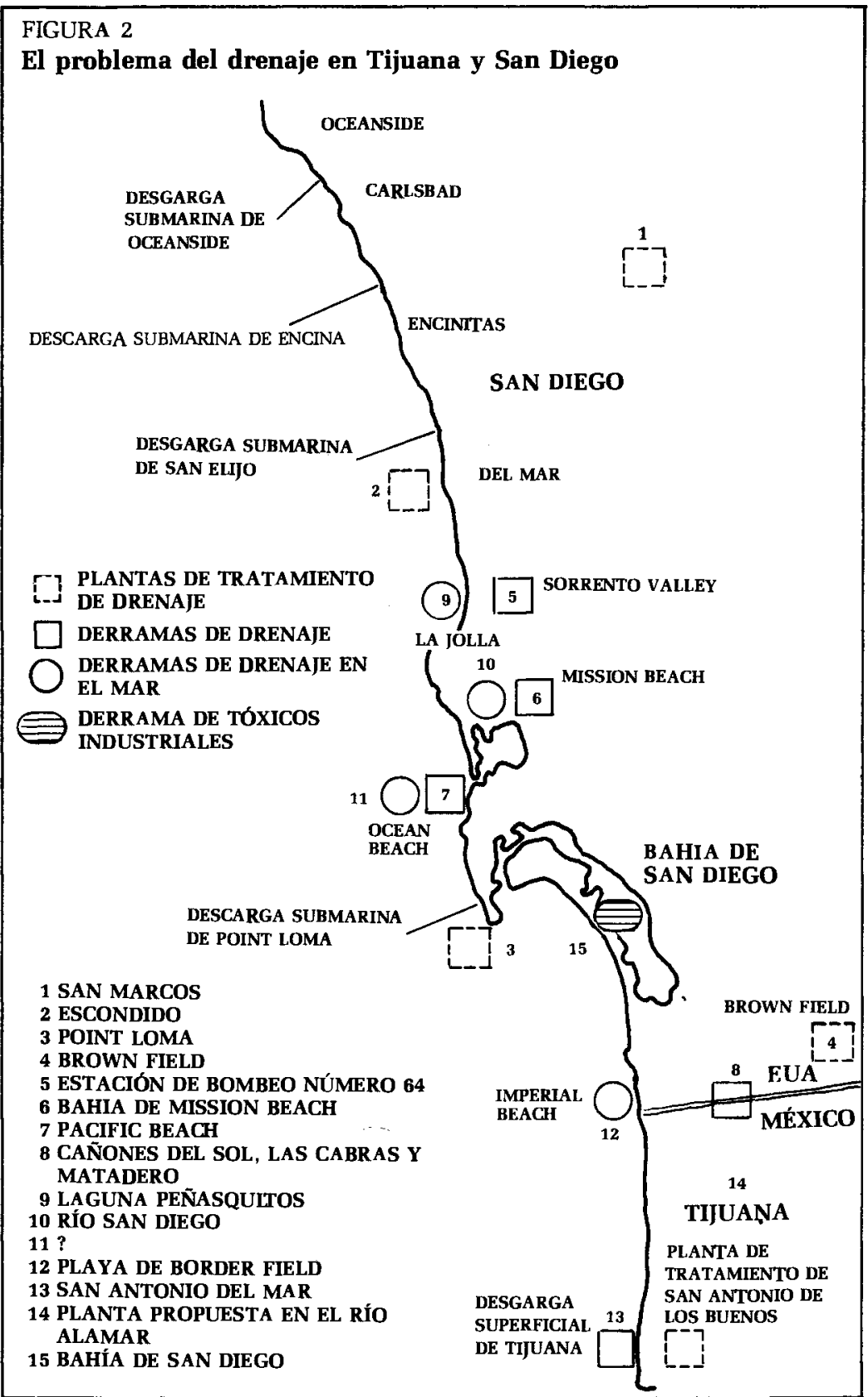


Las derramas de aguas servidas a lo largo de la avenida internacional y a través de los cañones que desembocan en la frontera han sido causa de la mayor parte de las críticas de Estados Unidos y San Diego hacia México. Una parte del problema lo constituyen las constantes rupturas en el colector central del sistema de Tijuana y las consecuentes derramas del efluente. El mal funcionamiento de las estaciones de bombeo agravaba con frecuencia las derramas de aguas servidas a través de la frontera. Esta situación fue recurrente durante la década de los setenta y se incrementó durante la primera mitad de los ochenta. En agosto de 1983, la ruptura de este colector y el mal funcionamiento de las bombas ocasionaron la derrama de cinco millones de galones de aguas servidas por día a través de la frontera (The San Diego Union, 10 de agosto de 1986). El efluente derramado inundó las propiedades colindantes con la frontera, el estuario del río Tijuana y las playas de Border Field e Imperial Beach. Esta fue la peor derrama del efluente de Tijuana.

La otra parte del problema en esta zona la constituyen las derramas de aguas servidas a través de los cañones que desembocan en la frontera. En este caso se trata del efluente proveniente de las colonias populares localizadas en las partes altas de Tijuana (colonia Francisco Villa, Obrera, Tenochtitlán, Cañón del Sol y otras), que no es colectado por el sistema de drenaje de la ciudad. El efluente sigue el dren natural del terreno y desemboca en la frontera en los cañones de El Sol, Las Cabras y El Matadero (véase la fig. 1). En la época de mayor flujo (1983-1985) se estimó que el efluente transportado por estos cañones era de cerca de cuatro millones de galones por día (mgd). Actualmente se estima que este flujo ha decrecido gracias a la extensión de la red del drenaje de Tijuana, aunque las autoridades de San Diego mencionan que el flujo se ha mantenido constante.

Por último, las descargas de aguas servidas en el litoral son, quizás, la parte central de la quejas de San Diego. Constantemente se argumentó que estas descargas constituían un enorme riesgo para la salud pública en San Diego, y que la contaminación generada podría afectar no sólo las playas próximas a la frontera en Imperial Beach, sino inclusive llegar hasta Coronado. Las descargas de aguas servidas en el litoral se realizaban en tres diferentes partes: en Playas de Tijuana y en San Antonio del Mar en el sur de la frontera, y en la desembocadura del río Tijuana en el norte de la frontera (véanse las figs. 1 y 2).

La deficiente operación de la pequeña planta de tratamiento en Playas de Tijuana ocasionaba la derrama de las aguas servidas de esta zona directamente en la playa. La descarga de cerca de $5 \mathrm{mgd}$ tenía lugar a 1.5 $\mathrm{km}$ de la frontera. El transporte de contaminantes hacia las playas de Border Field e Imperial Beach era frecuente y ocasionaba la cuarentena de cerca de $3 \mathrm{~km}$ de playa adyacentes a la frontera. Esta descarga se eliminó en 1986, al conectarse el drenaje de playas de Tijuana al canal de aleja- 
miento que transporta el drenaje de Tijuana a la planta de tratamiento. La descarga del efluente colectado y tratado de Tijuana, cerca de 20 mgd, se realiza actualmente $8 \mathrm{~km}$ al sur de la ciudad en la localidad de San Antonio del Mar.

La descargas de aguas servidas a través del río Tijuana no han sido controladas hasta el momento. Estas descargas son la principal fuente de contaminación en las playas de Imperial Beach. En la época de mayores descargas en 1984 y 1985, hasta $11 \mathrm{~km}$ de playa fueron declarados en cuarentena por altos registros de bacterias coliformes. A pesar de que en 1986 y 1987 decreció el flujo de drenaje a través del río Tijuana, en marzo de 1988 se reportó un fuerte incremento en este flujo (Los Angeles Times, 19 de abril de 1988). De acuerdo con las autoridades de esa ciudad, y con base en los registros del CILA estadunidense (el International Boundary and Water Commission, IBWC), este flujo de aguas servidas es actualmente superior a los 12 millones de galones por día (equivalente a cerca de la mitad del drenaje tratado en la planta de San Antonio del Mar). Debido a estas descargas, parte de las playas de Imperial Beach han sido declaradas una vez más en cuarentena con altos registros de bacterias coliformes. Otro daño ocasionado por esta misma fuente es la alteración al ecosistema del estuario del río Tijuana en su desembocadura al otro lado de la frontera (véase la fig. 1).

Las fallas en la operación de la estación de bombeo número 1 de Tijuana en febrero de 1988, y la consecuente derrama del efluente a través de la frontera ha contribuido a incrementar aún más la creciente tensión sobre este problema (véase la fig. 1).

\section{El drenaje de San Diego}

El estudio de la región Tijuana-San Diego muestra que en ambas ciudades existen fuentes contaminantes del litoral trasfronterizo. Al igual que Tijuana, San Diego es una ciudad de rápido crecimiento donde el drenaje es uno de los problemas urbanos más agudos. Fallas en el sistema de dre. naje del condado han ocasionado la derrama recurrente de aguas servidas en diversos puntos de la costa (véase la fig. 2).

San Diego cuenta con un sistema de drenaje centralizado que concentra en una sola planta de tratamiento $90 \%$ del efluente de todo el condado, equivalente a cerca de $170 \mathrm{mgd}$. Esta planta se localiza en Point Loma y su sistema de tratamiento es primario avanzado. Los problemas del drenaje de San Diego son similares a los de cualquier otra ciudad de rápido crecimiento con un sistema centralizado de drenaje. Por un lado, la ciudad ha crecido mucho más rápido de lo que se ha podido mejorar su sistema de drenaje; por otro, partes del sistema central son actualmente insu- 
ficientes para poder manejar el incremento en el efluente generado en esa ciudad.

Especialmente crítico es el caso de Mission Beach, un importante centro recreativo en donde frecuentes derramas de agua servidas han obligado a declarar en cuarentena partes de la bahía uno de cada cuatro días durante el periodo 1980-1986 (véase la fig. 2). ${ }^{7}$ Mission Beach es una de las partes de la ciudad que cuentan con tuberías viejas de concreto corroídas. La ciudad tenía noticia del deficiente estado de las tuberías en esta zona desde la mitad de la década de los cincuenta. No obstante, hasta 1980 no se llevó a cabo ninguna acción para remediar esto, a pesar de las frecuentes derramas del efluente. En 1980, un importante evento deportivo que debía tener lugar en la bahía de Mission Beach (una carrera internacional de remo), fue suspendido por derramas de agua servidas en la bahía. A partir de ese año el problema de Mission Beach se hizo público, pero no fue sino hasta 1985 cuando la ciudad empezó a cambiar las tuberías de concreto dañadas. De acuerdo con las autoridades de la ciudad, el remplazo de tuberías tardará siete años más (The Son Diego Union, 11 de febrero de 1986). Mientras tanto, es muy probable que sigan ocurriendo derramas de efluente en esta zona, de acuerdo con las declaraciones de las propias autoridades de la ciudad. ${ }^{8}$

Otra zona de derramas del drenaje de San Diego es el río San Diego y la playa estatal de Torrey Pines cerca de la laguna Peñasquitos. En este caso, las derramas son debidas a continuas fallas en la estación de bombeo número 64, que bombea el efluente recolectado en la parte norte del condado hacia la planta de tratamiento (The San Diego Union, 10 de agosto de 1986) (véase la fig. 2). Las autoridades de la ciudad tuvieron conocimiento de la falta de capacidad en esta bomba en 1981. En 1985, después de repetidas derramas, la ciudad de San Diego fue multada por el gobierno de California (California Regional Water Quality Control Board) con la cantidad de 341000 dólares. La ciudad empezó la construcción de una nueva estación de bombeo sólo después de esta multa. No obstante, las derramas del efluente continuaron. En noviembre de 1986, esta estación de bombeo derramó 1.5 millones de galones en Laguna Peñasquitos, una zona de protección ecológica en el norte de la ciudad, y en la playa estatal de Torrey Pines. ${ }^{9}$ Con ésta son 57 las derramas de esta estación en siete años.

\footnotetext{
7 Véanse por ejemplo las notas periodísticas en The San Diego Union, 9 de abril de 1985 , Los Angeles Times, 15 de junio de 1987 y The San Diego Union, 23 de marzo de 1986.

8 Entrevista televisada con Armand Campillo, director del Departamento de Agua de la Ciudad de San Diego, 17 de mayo de 1987.

${ }^{9}$ Las derramas de drenaje en Torrey Pines obligaron a declarar en cuarentena esta playa 63 días entre 1984 y 1986. Durante el mismo periodo, partes del río San Diego -Fashion Valley, Dog Beach, la desembocadura del río y otras- fueron declaradas tambien en cuarentena cuando menos 75 días (The San Diego Union, 10 de agosto de 1986).
} 
El 14 de enero de 1987, el California Regional Water Quality Control Board for San Diego propuso imponer una multa de 1.5 millones de dólares a la ciudad de San Diego por la derrama de aguas servidas de noviembre de 1986 (The San Diego Union, 16 de enero de 1987). Ésta es la multa más alta propuesta hasta ahora en el estado de California por este tipo de problema. Una nueva derrama de 4.5 millones de galones de drenaje, en enero de 1987, llevó al gobierno de California a proponer una multa adicional de 646800 dólares. En su informe, el California Regional Water Quality Control Board declara que las derramas son debidas a la negligencia de la ciudad para expandir la capacidad de la estación de bombeo y garantizar su correcta operación. Un mes después, en marzo de ese mismo año, sucedió una nueva derrama de 20 millones de galones de agua servidas en la misma estación (The San Diego Union, 6 de marzo de 1987). La ciudad de San Diego sólo ha pagado 200000 dólares en multas por derramas en esta estación. El resto de las multas quedaron supeditadas a que la ciudad cumpliera con el plazo pactado para la solución completa de este problema. El costo aproximado de estas obras fue de 8.7 millones de dólares, costo muy superior a los 2 millones programados inicialmente.

El problema de la estación de bombeo número 64 es muy similar al de la estación de bombeo número 1 en Tijuana. ${ }^{10}$ Tijuana fue objeto de severas críticas y dudas de las autoridades de San Diego sobre la capacidad de los mexicanos para operar un sistema de drenaje. Parece que cuesta trabajo reconocer los errores propios en el mismo campo. ${ }^{11}$

Otra fuente contaminante de la zona del litoral trasfronterizo la constituye la descarga de cerca de $\mathbf{1 7 0}$ millones de galones diarios de efluente tratado, que son vertidos al mar a través del emisor submarino de la planta de Point Loma (The San Diego Union, 10 de diciembre de 1986). San Diego proporciona un tratamiento primario de sedimentación a su efluente en esta planta antes de vertirlo al mar a través de un difusor submarino a dos y media millas de la costa (véase la fig. 2). Un reciente estudio de la Agencia de Protección Ambiental estadunidense ( $\mathrm{EPA}$ ) encontró que la descarga de Point Loma no cumple con los estándares federales para calidad de agua en grasas y aceites, sólidos suspendidos y sólidos sedimentables, turbulencia, oxígeno disuelto y bacterias coliformes, y que áreas recreativas cercanas al emisor frecuentemente no satisfacen los límites de

${ }^{10}$ El 27 de julio de 1988 la Agencia de Protección Ecológica estadunidense (EPA) demandó a la ciudad de San Diego por no cumplir con la legislación vigente del Acta para el Agua Limpia (Clean Water Act). La demanda menciona los problemas del drenaje en San Diego. Entre ellos, 1814 derramas de efluente en la costa y otros cuerpos de agua, y 92 descargas ilegales de lodos del tratamiento del efluente en Brown Field (cerca de la frontera) entre 1984 y 1986 (Los Angeles Times, 28 de julio de 1988).

11 Véanse por ejemplo las declaraciones de Ladin Delaney, director del California Regional Water Control Board for San Diego, en The San Diego Union el 10 de agosto de 1986, y en los Angeles Times el 7 de marzo de 1988). 
calidad de agua para coliformes fecales (Los Angeles Times, 4 de diciembre de 1986 y el San Diego Union del 5 de diciembre de 1986).

La comparación de la calidad del agua del drenaje crudo de San Diego y Tijuana realizada por Conway, Salgado y Álvarez (1987) muestra valores similares en demanda bioquímica de oxígeno, sólidos disueltos y suspendidos, nutrientes, altas concentraciones de coliformes fecales y bajos niveles de metales pesados. ${ }^{12}$ Esto concuerda con los valores reportados por Sañudo, Nishikawa y Rivera (1984) sobre concentración similar de metales trazas en el drenaje de las dos ciudades (plata, arsénico, cadmio, cromo, cobre, mercurio, níquel, plomo, selenio y zinc). No obstante, es importante tener en cuenta que San Diego descarga en el litoral ocho veces más efluente tratado que Tijuana, ${ }^{13}$ por lo que su emisión de contaminantes a través de estas descargas es considerablemente mayor. Debe considerarse, también, el tiempo que tardan los contaminantes en degradarse a través de la oxidación del efluente una vez en contacto con el mar. La literatura especializada menciona un periodo de oxidación de siete a ocho horas para el efluente descargado directamente sobre la superficie -el caso de Tijuana-, mientras que la oxidación de las descargas de un emisor submarino es de cerca de dos días - el caso de San Diego. ${ }^{14}$

Por último, cabe mencionar un reciente estudio sobre la calidad del agua en la bahía de San Diego realizado por el Servicio Nacional de Pesca Marina de Estados Unidos (The National Marine Fisheries Services). El estudio pone de manifiesto el alto nivel de contaminación en la bahía por productos altamente tóxicos y cancerígenos como PCB, prohibido en Estados Unidos desde 1979, y trybutil tin, considerado el químico más tóxico que jamás se ha puesto en el agua (Los Angeles Times, 3 de enero de 1987). En la bahía se han encontrado, además, altas concentraciones de cobre, plomo, cadmio, hidrocarburos aromáticos y moderadas concentraciones de DDT. La contaminación es causada por las descargas de una amplia gama de importantes empresas localizadas alrededor de la bahia (véase la fig. 2). Llama la atención la conclusión del estudio que clasifica a San Diego como la segunda bahía más contaminada en Norteamérica (The San Diego Union, 14 y 23 de enero de 1988). El California Regional Water Control Board for San Diego lleva a cabo actualmente un estudio más detallado sobre la contaminación de esta bahía.

${ }^{12}$ Estos mismos autores reportaron valores más bajos en estos contaminantes en el drenaje de Tijuana en las mediciones realizadas en el canal de alejamiento con respecto a las de la estación de bombeo número 1. Esto es un reflejo de la descomposición del efluente y la reducción de contaminantes en su paso hacia la costa.

${ }^{13}$ Cerca de 170 millones de galones por día son tratados y descargados en el océano en la planta de Point Loma (The San Diego Union, 10 de agosto de 1986).

${ }^{14}$ Entrevista con el doctor T. Hendricks, marzo de 1988 


\section{Las corrientes marinas}

Un aspecto de suma importancia en la discusión de la contaminación del litoral Tijuana-San Diego es el transporte de contaminantes a través de la frontera. Dos tipos de corrientes afectan el transporte de contaminantes debidos a las descargas de efluente en el litoral:

1) las corrientes superficiales inmediatamente adyacentes a la costa en una franja que varía de acuerdo con la topografía de la zona. Por lo general, se trata de una franja de unos cuantos cientos de metros próximos a la costa. Este tipo de corriente es responsable del transporte de las descargas superficiales del drenaje de Tijuana;

2) las corrientes superficiales y subsuperficiales, generalmente una franja entre los $500 \mathrm{~m}$ y los $6 \mathrm{~km}$ de la costa, que transportan los contaminantes de las descargas del emisor submarino de la planta de Point Loma o de cualquier otro futuro emisor submarino en la zona.

La información disponible sobre el flujo de estas corrientes es limitada; no obstante, permite establecer las siguientes consideraciones:

Los estudios sobre la corriente directamente adyacente a la costa son muy escasos. La mejor información se obtuvo del profesor Seymour del Instituto de Oceanografía de Scripps de la Universidad de California en San Diego, quien se dedica al estudio de la erosión de la costa en la zona comprendida entre Imperial Beach y la frontera. De acuerdo con el profesor Seymour, este tipo de corriente sigue un patrón de norte a sur, a partir de la desembocadura del río Tijuana en el estuario del mismo nombre. De hecho, el delta del río Tijuana forma un parteaguas en el flujo de la corriente. El acarreo de sedimentos por el río y la formación del delta modifican la topografía del área directamente adyacente a la costa. A partir de ese punto, la corriente fluye por un lado hacia el sur y por otro hacia el norte-(véase la fig. 1). Esto quiere decir que la descarga superficial de efluente de la planta de San Antonio de los Buenos al sur de Tijuana, no afecta las costas de San Diego. El efluente descargado en la costa tiende a ser transportado hacia el sur, como lo demuestran los resultados de la investigación realizada por la Secretaría de Marina en 1986 (Morales, 1987). De acuerdo con estos datos, los registros de coliformes fecales a lo largo de la costa decrecen hacia el norte y crecen hacia el sur, a partir de la descarga de esta planta.

Sin embargo, las descargas de efluente sobre la costa a través del río Tijuana (el caso de las recientes derramas) son transportadas hacia el norte, afectando las playas de Imperial Beach. El transporte de los contaminantes dependerá del volumen de la descarga y de la intensidad del oleaje en la zona.

Las corrientes comprendidas en la franja amplia de $6 \mathrm{~km}$ de la costa tienen un patrón de corriente de norte a sur. En su estudio sobre este tipo de corrientes, Luis Álvarez (1986) del Centro de Investigación, Ciencias 
y Estudios Superiores de Ensenada (CICESE), demuestra con evaluaciones mensuales durante 1985 un flujo de norte a sur en la mayor parte del año (véase la fig. 1). Las excepciones son los meses de septiembre y noviembre en que la corriente cambia de dirección hacia el norte. Álvarez menciona que aunque el flujo de sur a norte es más cercano a la costa, su capacicaad de transporte y factor de dilución son menores que cuando la corriente fluye de norte a sur.

El International Boundary and Water Commission (IBWC) reportó también un flujo constante de norte a sur durante la mayor parte de 1986 en la corriente cercana a la costa entre San Diego y Tijuana. ${ }^{15}$

Por su parte, el doctor Tareah Hendricks del Instituto de Oceanografía de Scripps, quien ha estudiado el patrón de corrientes en esta zona por varios años, reporta un flujo relativamente uniforme de norte a sur durante 1986-1987 (Hendricks, 1987) (véase la fig. 1). Sin embargo, menciona la posible influencia del fenómeno natural sobre el Pacífico conocido como "El Niño". El doctor Hendricks enfatiza la necesidad de llevar a cabo estudios detallados con el objeto de poder determinar con exactitud el posible transporte de contaminantes debidos a una descarga de drenaje submarina. Para el caso de Point Loma, la información del doctor Hendricks (1977) muestra un movimiento de la corriente subtermohalina costa arriba durante 290 días de 1977 , con una velocidad de $3 \mathrm{~cm} / \mathrm{seg}$. Sin embargo, debe estudiarse si el movimiento de esta corriente mantiene actualmente la misma dirección. De igual forma, debe estudiarse cuál es la dirección del transporte de la descarga del futuro emisor submarino que la ciudad de San Diego planea construir en el límite internacional entre México y Estados Unidos (véase la fig. 1). Hasta el momento no se ha definido de que forma se verá afectada esta descarga a través del patrón de corrientes en esa zona.

Esta información es de vital importancia en la definición del impacto de las descargas del efluente de Tijuana en San Diego y viceversa. El flujo de corrientes de norte a sur implica que las descargas de Tijuana en San Antonio del Mar, $8 \mathrm{kms}$ al sur de Tijuana, no afectan las playas de San Diego. Los altos valores en coliformes fecales registrados en las playas de Border Field hasta 1986, se debieron en su mayor parte a las descargas de drenaje en Playas de Tijuana cerca de la frontera. Una vez controladas estas descargas en ese año, se eliminó este problema. Sin embargo, se mantiene el de la contaminación de las playas de Imperial Beach por las descargas de efluente de Tijuana a través del río Tijuana. Por último, queda por definir el posible impacto en México de las futuras descargas en el limite internacional programadas por San Diego.

${ }^{15}$ Declaraciones del Dr. Gunashi, director del IBwc, ante el San Diego Sewage Task Force, 7 de junio de 1987. 
Los resultados de estos estudios son también relevantes como factor de negociación para México: Ayudan a cambiar la imagen, difundida por los medios de comunicación en San Diego, sobre la enorme amenaza que representa para las playas de esa ciudad, el drenaje de Tijuana y cualquier falla en la operación de la planta de tratamiento de San Antonio del Mar.

\section{El sistema defensivo}

Otro aspecto a considerar sobre el problema del drenaje en Tijuana y San Diego es el proyecto y construcción de un "sistema defensivo" en San Diego próximo a la frontera. El objetivo de este proyecto es capturar el efluente de Tijuana que cruza la frontera para ser bombeado de regreso a Tijuana. El apoyo legal del sistema defensivo es la minuta 264 , que modifica al tratado de 1944 , y a través de la cual México se compromete a recibir de regreso su efluente. El sistema defensivo consta de tres diferentes proyectos.

El primero corresponde a la habilitación y construcción de canales por parte del IBWC para colectar las aguas servidas el cual cruza la frontera en la zona de los cañones (de El Sol, Las Cabras y El Matadero). El efluente recolectado será bombeado de regreso a México. La primera parte de estos canales se terminó en noviembre de 1987 y la segunda en abril de 1988. El costo de cada una de estas partes fue de aproximadamente 400000 dólares (The San Diego Union, 17 de octubre de 1987).

El segundo proyecto del sistema defensivo lo constituyen los proyectos de la ciudad de San Diego conocidos como 1D y 7C (véase la fig.1). El proyecto 1D surgió en 1983 y el costo de su diseño (400 000 dólares) fue finänciado por el estado de California. La idea del proyecto es similar a la del caso anterior; recoger el efluente que cruza la frontera por los cañones que desembocan en la avenida internacional, con el fin de enviarlo de regreso por gravedad a la estación de bombeo número 1 y al sistema de drenaje de Tijuana. La capacidad del proyecto es de 6 mgd (Ciudad de San Diego, 1987). Actualmente la construcción del proyecto se encuentra detenida por la habilitación de los canales del IBWC que realizan la misma función programada para el proyecto $1 \mathrm{D}$, y por la nueva propuesta de un proyecto mayor en la misma zona conocido como the big pipe.

El proyecto 7C surgió en 1984 con la idea de complementar la función del proyecto 1D (véase la fig. 1). Su idea básica es establecer un sistema de refuerzo (backup) de la operación de la estación de bombeo número 1 en Tijuana. El proyecto recogerá el eflúente de Tijuana derramado a través de la frontera en caso de fallas con la estación de bombeo número 1. El efluente será bombeado de regreso a México cerca de playas de Tijuana para canalizarlo a la planta de tratamiento en San Antonio del Mar. 
La capacidad del proyecto es de $34 \mathrm{mgd}$, equivalente a la capacidad del canal de alejamiento del sistema actual de drenaje de Tijuana. Al igual que en el caso del proyecto $1 \mathrm{D}$, la construcción del proyecto $7 \mathrm{C}$ dependerá de la aprobación y construcción del proyecto big pipe. ${ }^{16}$

La tercera y más reciente fase del sistema defensivo de San Diego está formado por el proyecto big pipe (véase la fig. 1). La idea básica de este proyecto es similar a la de los anteriores: recolectar el efluente de Tijuana que cruza la frontera para enviarlo de regreso. Sin embargo, existen importantes diferencias: el big pipe ha sido presentado por las autoridades de San Diego como una solución de largo alcance a la inaceptable contaminación del estuario del río Tijuana y las playas de South Bay por las derramas de Tijuana (Buttle/Roach Group, 1988). De acuerdo con las autoridades de San Diego, este flujo es actualmente casi $5 \mathrm{mgd}$ en la zona de cañones. El proyecto está pensado para captar también el incremento futuro de aguas servidas de Tijuana, con una capacidad de $100 \mathrm{mgd}$. Se espera que el proyecto sea también una solución a la posible contaminación del estuario del río Tijuana a través del río del mismo nombre, que transporta las actuales derramas sin tratar, o por las futuras descargas de efluente tratado por la planta del Alamar. ${ }^{17}$

El proyecto consiste en un canal con una capacidad de $340 \mathrm{mgd}$ y un costo aproximado de 34.5 millones de dólares, el cual recoge el efluente derramado a lo largo de la frontera y por el río Tijuana para bombearlo de regreso a Tijuana (Los Angeles Times, 8 de marzo de 1988). Las autoridades de la ciudad de San Diego lo conciben como una excelente posibilidad de cooperación entre la ciudad de San Diego, la Agencia de Protección Ambiental estadunidense, el U.S. Army Corps of Engineers y el State Water Resources Control Board. Hasta ahora no se ha incluido a la parte mexicana para participar en la discusión de este proyecto.

Para las autoridades de San Diego, la rehabilitación de la zona de South Bay le permitirá recuperar un ingreso anual estimado de 108 millones de dólares por turismo y actividades recreativas, y una plusvalía estimada de 85 millones de dólares en las propiedades locales. De acuerdo con las autoridades de la ciudad, éstas son las pérdidas locales ocasionadas por las recurrentes derramas de las aguas servidas de Tijuana (Buttler/Roach group, 1988).

El proyecto del big pipe tiene una relación directa con el proyecto de

${ }^{16}$ Entrevista con Peter Silva, ingeniero en el Departamento de Servicios de Agua, División de Proyectos Especiales, de la ciudad de San Diego, y antiguo representante del IBwc en San Diego, 4 de abril de 1988.

${ }^{17}$ El ecosistema del estuario es muy frágil. Cualquier alteración en sus condiciones de equilibrio puede causar la perdida del ecosistema. En este caso, el equilibrio entre la intrusión de agua salada y agua dulce es clave para la existencia del estuario. La descarga de efluente tratado en el río Tijuana romperia las condiciones de equilibrio del estuario. 
una planta de tratamiento en South Bay. ${ }^{18}$ San Diego debe ampliar el tratamiento a sus aguas servidas de primario avanzado a secundario antes de verterlo al mar; así cumplirá con las nuevas disposiciones del acta federal para el agua limpia (Clean Water Act). Esto requiere la construcción de una o varias nuevas plantas de tratamiento en ese condado. A pesar del reciente inicio de la planeación de un nuevo sistema de drenaje en San Diego en marzo de 1988, existe un amplio consenso entre las autoridades de esta ciudad de que una de las nuevas plantas de tratamiento se construirá en el sur del condado, justo en la frontera y prácticamente en el mismo lugar seleccionado para la planta internacional en 1984 (The San Diego Union, 13 de marzo de 1988) (véase la fig. 1).

La ciudad de San Diego tiene actualmente más de 21 posibles escenarios para la ampliación del sistema de drenaje de San Diego, y la construcción de una planta en la frontera con México aparece en 18 de los 21 considerados (The San Diego Union, 13 de abril de 1988). Dos de ellos son señalados como los más probables (véase la fig. 2):

1) un sistema con dos plantas de tratamiento, una en South Bay y otra en Point Loma. En este caso, la planta de South Bay tendría una capacidad cercana a los $240 \mathrm{mgd}$, y la planta de Point Loma de $120 \mathrm{mgd}$;

2) un sistema con tres plantas: una en la parte norte del condado, otra en Point Loma y una planta en South Bay. En este escenario, la carga total del sistema se repartiría entre las tres plantas. La planta de South Bay tendría una capacidad aproximada de $100 \mathrm{mgd}$.

Dentro del resto de las alternativas presentadas, existe también la de concentrar la totalidad del efluente de San Diego en la planta de South Bay, con una capacidad de $360 \mathrm{mgd}$. Bajo esta alternativa, San Diego trataría la totalidad de sus aguas servidas justo en la frontera con México.

La planta de tratamiento en South Bay, en cualquiera de los dos escenarios, incluye un emisor submarino para la descarga del efluente tratado (véase la fig. 1). Las autoridades de San Diego programan que el proyecto big pipe forme parte del sistema de la planta de South Bay, ${ }^{19}$ conectando la planta con el emisor submarino para descargar el efluente en el mar. Como parte de esta alternativa, se propone cambiar la idea inicial del big pipe de un solo canal por dos drenes, uno para transportar efluente de Tijuana y otro para transportar el tratado en la planta de South Bay (Los Angeles Times, 19 de abril de 1988). Dentro de los beneficios de este es-

${ }^{18}$ El proyecto big pipe ha sido objeto de críticas por parte de los representantes electos de South Bay, quienes temen que la construcción del dren conducirá irremediablemente a la construcción de una superplanta de drenaje en esta parte de la ciudad (The San Diego Union, 17 de marzo de 1988).

${ }^{19}$ Aunque las mismas autoridades declaran que la construcción del dren no implica necesariamente la construcción de la planta de tratamiento (véanse las declaraciones en el San Diego Union, febrero de 1988). 
quema se menciona el reúso de agua. De acuerdo con diversas autoridades de San Diego, esta ciudad estaría dispuesta a ofrecer el agua tratada de esta planta para su reúso en México. Esto es una alternativa que podría estudiarse, en particular tomando en cuenta las condiciones semidesérticas de Baja California.

Por otro lado, la Agencia de Protección Ambiental (EPA) estadunidense considera actualmente la posibilidad de que la planta de South Bay sea una planta binacional. En este proyecto, dicha planta tendría capacidad para tratar el efluente de Tijuana. Esta misma idea es considerada por las autoridades de San Diego, quienes tienen un doble interés en el proyecto. Además de la vieja idea de construir la planta binacional como la mejor alternativa para solucionar el problema de Tijuana, su carácter binacional ayudaría a San Diego a lograr una excepción para que esta planta no cumpla con los requisitos del Acta Federal para Agua Limpia (Clean Water Act) de tratamiento secundario en el tiempo límite fijado. San Diego tiene actualmente problemas para cumplir con estas nuevas disposiciones federales ${ }^{20}$ La EPA concedería esta excepción a San Diego (sección $5.10 \mathrm{del}$ Clean Water Act de 1987) sólo para el efluente de Tijuana que la planta de South Bay llegara a tratar. En este escenario, el efluente de Tijuana tratado en South Bay no requeriría tener un tratamiento secundario antes de ser vertido al mar. Éste es un factor que debe considerarse si en algún momento se decide negociar la posibilidad de construir una planta binacional. México podría reducir su inversión financiera en este proyecto argumentando que, de acuerdo con la legislación estadunidense, sus aguas servidas sólo requieren un tratamiento primario avanzado.

Una propuesta para la construcción de la planta binacional fue presentada a México a través del IBWC y el Comite de Límites y Aguas (CiLA) de la Secretaría de Relaciones Exteriores. La EPA solicitó de México una carta de interés sobre su participación en este proyecto, pero hasta este momento no existe una respuesta oficial. ${ }^{21}$

\section{Implicaciones para México}

Los proyectos de expansión del drenaje de San Diego tienen importantes implicaciones para México. De seguirse el esquema de sólo dos plantas en San Diego, una en South Bay y la otra en Point Loma, la planta en South Bay tendría una capacidad de $240 \mathrm{mgd}$. Es decir, $50 \%$ superior a la capacidad actual de Point Loma, que trata $90 \%$ del efluente en ese condado.

${ }^{20} \mathrm{El}$ Clean Water Act demanda un tratamiento secundario en el efluente de las plantas de tratamiento de drenaje antes de su descarga en el mar.

${ }^{21}$ Entrevista con Richard Reavis, coordinador fronterizo (Border Coordinator) de la EPA en San Diego (28 de marzo de 1988). 
En este esquema se concentraría la mayor parte del efluente de San Diego justo en la frontera con México. Esto le permite a San Diego evitar el conflicto político con las otras comunidades del condado, que se rehúsan a aceptar en su jurisdicción una planta de tratamiento de drenaje. ${ }^{22}$ Las características de South Bay - una de las zonas más pobres del condado y donde se concentra la mayor parte de la población de origen mexicanola presentan como la zona de menor conflicto político. Jim Bates el representante electo de esta zona ante el congreso estatal de California, ha protestado por la construcción de esta planta (The Son Diego Union, febrero de 1988). No obstante, en San Diego existe un amplio consenso en favor de llevar a cabo su construcción en esta zona. Un aspecto que refuerza esta hipótesis es la propuesta del canal colector conocido como big pipe. Es indudable que este proyecto tiene una relación directa con la superplanta de $\mathbf{2 4 0} \mathrm{mgd}$ en la frontera. No existe otra explicación posible para la enorme capacidad programada para el canal $(340 \mathrm{mgd})$ de los cuales $240 \mathrm{mgd}$ se usarían para conectar la planta con el emisor submarino y 100 mgd colectarían el tratado o crudo de Tijuana que cruzara la frontera, para bombearlo de regreso a México.

Aunque la construcción del proyecto big pipe es aún incierta, debido a que San Diego no ha logrado reunir los fondos necesarios para llevarla a cabo, México debe pedir una discusión amplia de estos proyectos dentro del marco de la negociación binacional. Una superplanta de la magnitud planeada para South Bay podría tener importantes consecuencias en Tijuana y en el litoral mexicano. México debe estudiar el posible impacto de su construcción y operación en la zona adyacente al área urbana de Tijuana, sobre todo en el caso de una emergencia por fallas en su operación. Debe estudiar también el impacto de la futura descarga submarina de esta planta, justo en el límite internacional entre México y Estados Unidos, y el posible transporte de contaminantes hacia el sur.

Aun en el caso de que el proyecto big pipe no llegara a construirse, su sola propuesta tiene un impacto en la negociación binacional. Este proyecto representa la desproporcionada desconfianza de las autoridades de San Diego en la capacidad y voluntad de México para resolver el problema del drenaje de Tijuana. Aunque esto mismo se refleja en el resto de los proyectos que integran el sistema defensivo de San Diego, el big pipe es el extremo de la desconfianza. Su capacidad prevista para captar efluente de Tijuana, $100 \mathrm{mgd}$, es cerca de tres veces el total de las aguas servidas actuales de Tijuana. En este esquema, San Diego prácticamente presupo-

${ }^{22}$ Esto es un conflicto permanente en San Diego. Las comunidades se rehúsan a aceptar cualquier tipo de instalación para el tratamiento de desechos en su área. Por ejemplo, el proyecto para la construcción de una planta recicladora de basura originó largas protestas de los habitantes en la zona de Escondido, y fue objeto de una larga lucha política entre los habitantes de esta zona y las autoridades de la ciudad. 
ne que la totalidad del efluente de Tijuana será derramado a través de la frontera cuando menos durante los próximos 10 años.

Sin duda, el retraso en la construcción de la planta del Alamar y las fallas en el periodo de pruebas de la planta de San Antonio del Mar han favorecido la desconfianza de San Diego. Lo mismo sucede con las nuevas derramas de aguas servidas que cruzan la frontera en el río Tijuana. A pesar de que estos aspectos mantienen vivo el conflicto del drenaje, no justifican el grado de desconfianza mostrado por las autoridades de San Diego. En especial, no se justifica que San Diego no reconozca el esfuerzo y compromiso mostrado por México para solucionar el problema mediante la construcción de la planta en San Antonio del Mar y la ampliación de la red de drenaje de Tijuana.

La desconfianza en la negociación binacional no ayuda a lograr acuerdos de cooperación; por el contario, genera obstáculos. Sin duda el más grave es la duplicidad de inversiones en cada país para tratar de resolver el mismo problema, pero con diferente perspectiva. Mientras que México trata de establecer una solución permanente, San Diego procura sólo defenderse regresando el efluente a Tijuana. Ésta es la lógica del sistema defensivo. Su construcción costará cerca de cuatro veces lo que México ha invertido hasta ahora en la construcción de la planta de San Antonio del Mar en el periodo de la peor crisis económica del país. ${ }^{23}$

Es difícil predecir el impacto de la actitud de desconfianza de San Diego en la nueva ronda de la negociación binacional para la solución del problema de Tijuana. El peor escenario sería la repetición de la situación de 1984 y 1985, de tensas negociaciones que obligaron a México a buscar una solución propia al problema fuera de cualquier posibilidad de cooperación trasfronteriza. Aunque esta posibilidad existe, la situación en 1988 es diferente a la de 1984-1985. México ha demostrado un compromiso para solucionar este problema que contrasta con la poca atención que se le otorgó durante los años anteriores al acuerdo binacional. Otra diferencia importante es la información disponible y el conocimiento actual sobre el impacto real de las derramas del efluente de Tijuana en San Diego. El estudio del patrón de corrientes superficiales adyacente a la costa muestra un flujo predominante la mayor parte del año de norte a sur. ${ }^{24}$ Por primera vez México tiene argumentos para modificar la imagen que pretende pre-

${ }^{23}$ La solución del problema de Tijuana ha sido la prioridad más alta para la Sedue por la inversión de recursos y la atención otorgada a su solución de 1984 a 1988. Entrevista con Javier Valdez, director de Proyectos de la Dirección de Infraestructura Urbana de la Sedue, 9 de enero de 1988.

${ }^{24}$ Esto quedó demostrado durante la reparación de las lagunas de la planta de San Antonio de los Buenos de octubre de 1987 a febrero de 1988. En estos meses, casi la totalidad del efluente de Tijuana fue vertido al mar sin tratamiento previo. El transporte de las aguas servidas hacia el sur fue evidente. El muestreo de la calidad del agua en las playas de San Diego no mostró ningun cambio durante este periodo. 
sentarlo como la fuente contaminante y la peor amenaza para las playas de San Diego.

Por el contrario, la información actual permite definir que la contaminación en el litoral entre Tijuana y San Diego es debida a fuentes contaminantes en los dos lados de la frontera. Inclusive, queda por definirse la forma como México es afectado por el transporte de contaminantes originados en San Diego. Debe tenerse en cuenta que parte de estos contaminantes son residuos industriales de difícil control por un tratamiento convencional de drenaje, y de difícil dilución una vez depositados en el mar. Estos contaminantes constituyen un riesgo para el litoral trasfronterizo que debe estudiarse y discutirse en el marco de la negociación binacional.

El mejor escenario para las dos ciudades es, quizás, lograr la cooperación necesaria para construir una planta binacional. En contraste con la experiencia negativa de 1984, cuando la propuesta de una planta binacional se realizó fuera de toda posibilidad para lograr la participación de México, ${ }^{25}$ el resurgimiento de esta idea tiene lugar en mejores condicioñes. La expansión del sistema de drenaje de San Diego incluirá, muy probablemente, la construcción de una planta en South Bay, justo en la frontera con México. Por su lado, Tijuana debe construir la planta del Alamar en el corto plazo. Podría suponerse que sería de interés para las dos ciudades unir fuerzas y construir una planta binacional. Sin embargo, un aspecto clave para lograr una participación conjunta será la división de costos de construcción y operación entre los dos países. México no estaría en posibilidad de pagar más de lo que tiene previsto para la construcción de la planta del Alamar. Si Estados Unidos negocia la construcción de la planta binacional con una actitud flexible y acepta las limitantes económicas de México, el proyecto tiene posibilidades de realizarse.

Por su parte, México podría estudiar alternativas para reducir su inversión en el proyecto. Un tipo de participación financiera en el proyecto podría ser a través del pago en especie. Por ejemplo, México puede vender la electricidad para la operación de la planta al mismo precio que la vende actualmente a la San Diego Gas and Electric Co. La diferencia entre el costo de lo que la ciudad de San Diego pagaría a la San Diego Gas and Electric Co. (una compañía privada) por la electricidad y lo que se le pagaría a México, puede bonificarse a este último como parte de su participación financiera en el proyecto. ${ }^{26}$ Otro tipo de intercambio en especie

25 México debía pagar $60 \%-400$ millones de dolares - del costo total de la planta y cerca de 6 millones de dólares por su operación anual.

26 El precio de la electricidad en San Diego es uno de los más altos en la Unión Americana. Actualmente, $10 \%$ de la electricidad de San Diego proviene de Baja California, en particular de la geotermia de Cerro Prieto. A través de un contrato por 10 años entre la San Diego Gas and Electric Co. y la Comisión Federal de Electricidad, México vende 170 megawatts a esa ciudad. 
podría ser que México proporcione materiales y mano de obra para la construcción de la planta.

Por último, México debe estudiar la posibilidad de modificar su estrategia de acción durante la negociación binacional. Una alternativa podría ser la creación de alianzas con grupos estadunidenses que están a favor de lograr una solución al problema de Tijuana a través de la cooperación entre los dos países. Entre este tipo de grupos se incluye a los ambientalistas, algunos de ellos con un reconocido peso a nivel nacional en Estados Unidos -el Sierra Club y el Environmental Defense Fund- y a grupos locales, pero también a un diverso tipo de organizaciones e inclusive coaliciones políticas. La actitud de algunos de estos grupos ha sido favorable a prestar ayuda financiera a México para la solución del problema del drenaje de Tijuana y Mexicali, y a reducir su participación financiera en posibles proyectos conjuntos. Por ejemplo, gracias a la iniciativa de estos grupos, el congreso de California aprobó en 1987 una propuesta para liberar 150 millones de dólares destinados a la solución de problemas de contaminación trasfronteriza entre California y Baja California (The San Diego Union, 8 de abril de 1987; 14 de julio de 1987). Aunque la propuesta fue bloqueada por el gobernador de California, argumentando que se trataba de un asunto federal, y por lo tanto debería ser resuelto con dinero de la federación, existe consenso entre diversos congresistas de ese estado en favor de volver a presentar la propuesta en 1988, con mejores posibilidades de aprobarse.

Por otro lado, parece que la administración Reagan está dispuesta a modificar su posición poco flexible de 1984 y 1985 en la nueva ronda de negociaciones sobre el drenaje de Tijuana. En principio, la administración federal estadunidense ha aprobado cierta ayuda para la planeación y construcción del sistema defensivo en San Diego. Sin embargo, de acuerdo con algunas autoridades de la EPA, esta ayuda podría ampliarse para ayudar a México a participar en la posible construcción de una planta binacional.

Una estrategia que podría estudiarse es la de realizar lobbing en los congresos de Washington y Sacramento en favor de México. Existen resultados positivos que demuestran los beneficios de estas acciones. En el mes de abril de 1988, México logró detener una propuesta de varios congresistas estadunidenses para suspenderle ayuda finaciera bajo el argumento de una baja participación de nuestro país en la lucha contra el narcotráfico. Acciones semejantes pueden tener también un impacto positivo en la solución de los conflictos ambientales entre los dos países, y en particular en la solución del problema del drenaje de Tijuana. En este sentido, la alianza con grupos como los arriba mencionados será un elemento clave para poder lograr el impacto requerido en la administración Reagan y en el congreso en Washington y Sacramento.

México no debe subestimar el poder de presión de estos grupos den- 
tro de las estructuras políticas de Estados Unidos, y debe procurar una comunicación directa con ellos en busca de intereses comunes. Por ejemplo, un buen número de grupos ecologistas vería como un excelente intercambio la participación de México en la preservación del estuario del río Tijuana. En el largo plazo la construcción de la planta binacional ofrece la mejor alternativa de solución para los dos lados de la frontera. México puede ofrecer el compromiso de controlar en el corto plazo el flujo de agua en el río, servidas o tratadas, a un nivel aceptable que no ponga en peligro el equilibrio ecológico del estuario. A cambio, estos grupos participarían en una amplia campaña de presión a nivel local, estatal y federal en Estados Unidos, en favor de reducir la participación financiera en la contrucción de la planta binacional.

México podría obtener dos beneficios importantes de este tipo de alianzas: 1) limitar su participación financiera en la construcción de obras para la solución del problema de acuerdo a su capacidad de inversión; 2) influir en la posición estadunidense durante la negociación binacional en pro de una mayor cooperación. La acción conjunta de grupos en San Diego y la parte mexicana en defensa de intereses comunes, puede tener un importante impacto en la toma de decisiones de los congresos de California y Washington y de la administración Reagan.

De la actitud adoptada por ambos gobiernos, así como la de todos los grupos interesados en la solución del problema de Tijuana, depende establecer un precedente importante de cooperación binacional para la solución de otros problemas de contaminación trasfronteriza entre México y Estados Unidos. Ésta es, quizás, la última oportunidad en mucho tiempo para establecer este tipo de cooperación en la región de Tijuana-San Diego.

\section{Bibliografía}

Álvarez, G. Luis (1986), “Estudio oceanográfico sobre dispersión en las aguas costeras de Tijuana: programa de observaciones y resultados preliminares", en Juan Álvarez y Víctor Castillo (coords.), Ecología y frontera, México, Universidad Autónoma de Baja California.

Buttler/Roach Group (1988), Environmental Impact Report, South Bay Outfall-Phase I. Prepared for the City of San Diego, Water Utilities Department, San Diego.

Carroll, John (1986), "Water Resource Management as an Issue in Environmental Diplomacy", en Natural Resources Journal, New Mexico, The University of New Mexico School of Law, vol. 26, núm. 2".

Conway, John, Juan Álvarez y Héctor Salgado (1987), “'Tijuana and Tecate Border Monitoring Program in the Tijuana River Drainage Basin", mimeo.

Hendricks, Tareah (1977), "Costal Currents", en Costal Water Research Project Annual Report 1977, California.

y Niels Christensen (1987), "Modeling of Flow Fields and Effluent Trajectories in the San Diego Bight", mimeo.

Hill, Martin (1985), “Deadly Horizons', en San Diego Magazine, marzo. 
Meyer, E.L. (1983), "History of Sewarage Facilities Serving the City of Tijuana, Baja California, Mexico", California Regional Water Control Board, San Diego Region, mimeo.

Morales, Rafael (1987), "Apoyo al proyecto de evaluación de la contaminación en Playas de Tijuana, B.C.", Secretaría de Marina, mimeo.

Morales, Rafael y Carlos Romero (1986), "Evaluación de la contaminación en Playas de Tijuana, B.C.; reporte preliminar", en Juan Álvarez y Víctor Castillo, (coords.), Ecología y frontera, México, Universidad Autónoma de Baja California.

Mumme, Stephen y Joseph Nalven (1986), "Managing the Border Environment: Advances, Issues and Options", ponencia presentada en la tercera reunión ANUIES-PROFMEx, Santa Fe, Nuevo Mexico, 16-18 de abril.

Nalven, Joseph (1986), "Social Aspects of Trasboundary Environmental Cooperation”, en Juan Álvarez y Víctor Castillo (coords.), Ecología y frontera, México, Universidad Autónoma de Baja California.

Ojeda, Mario (1983), "The Future of Relations between Mexico and the United States", en Clark Reynolds y Carlos Tello (eds.), U.S.-Mexico Relations: Economic and Social Aspects, Standford, Standford University Press.

Sañudo, Sergio, Katsuo Nishikawa e Ignacio Rivera Duarte (1984), "Características de las aguas residuales de la ciudad de Tijuana, B.C.", mimeo.

Sánchez, Roberto (1988), "Las relaciones binacionales como un marco conceptual en el análisis de los problemas ambientales transfronterizos entre México y Estados Unidos", en Albert Utton y Emilio Marroquín (comps.), Una fronte.ra: dos países, México, ANUIEs.

Waisman, Alan (1984), "A Matter of Jurisdiction", en Atlantic Monthly, julio.

\section{Referencias periodísticas}

Abrams, Michael, "Bay's Water Ski Quarantined”, en The San Diego Union, 23 de marzo de 1986.

Bailey, Eric, "Big Pipe to Return Tijuana Sewage Given Initial Council O.K.", en Los Angeles Times, 8 de marzo de 1988.

Bunting, K., "State May Aid Mexican Sewage Cleanup", en Los Angeles Times, 4 de julio de 1986.

Dewey, Jackie, "To Stop Sewage Flow Across Border, Pressure on Mexico Must Continue", en Los Angeles Times, 2 de junio de 1985.

Fay, Rimmon, "California's Littoral of Toxic Waste", en Los Angeles Times, 6 de julio de 1986.

Flynn, George, "Agreement on Sewage Extended", en The San Diego Union, 15 de diciembre de 1986.

Frammolino, Ralph, "U.S. and State Sue City over Sewage", en Los Angeles Times, 28 de julio de 1988.

Gandelman, Joe, "EPA Fund New Sewage Project", en The San Diego Union, 17 de octubre de 1987.

"Treatment Plant Shut in Tijuana", en The San Diego Union, 6 de noviembre de 1987.

Hudon, Ken, "Tijuana Sewage Called Threat to Estuarine Sanctuary Plans", en The San Diego Union, 26 de septiembre de 1985.

Larue, Steve, "Nature Confines Bay's Sewage Spill", en The San Diego Union, 9 de abril de 1985.

, "City Gets Warning on Sewer Spills", en The San Diego Union, 11 de febrero de 1986. 
"Beach Pollution Picture is Murky for the Year 2000", en The San Diego Union, 10 de agosto de 1986.

"'Sewage Gushes in City's Worst Spill", en The San Diego Union, 6 de marzo de 1987.

"Wildlife species have stake in battle over the bay", en The San Diego Union, 11 de agosto de 1987.

“'Bay Found Plagued by 2 Pollutants", en The San Diego Union, 14 de enero de 1988.

"'Greater Anti-Pollution Effort urged for S.D. Bay", en The San Diego Union, 23 de enero de 1988.

Lubrano, Gina, "Sewage Spill May Cost City \$1.5 Million”, en The San Diego Union, 16 de enero de 1987.

Miller, Marjorie, "Mexico Offers to Build 2 Sewage Plants", en Los Angeles Times, 5 de febrero de 1985.

Sánchez, Raymond, "EPA Will Study Border Sewage Project", en Los Angeles Times, 19 de abril de 1988.

Scott, Janny, "Study Finds High Level of Toxics in S.D. Bay", en Los Angeles Times, 3 de enero de 1987.

Smolens, Michael, “\$150 Million Bond Plan to Fight Border Sewage Gains", en The San Diego Union, 8 de abril de 1987.

"'State Panel Approves Sewage Bill", en The San Diego Union, 14 de julio de 1987.

Warren, Jenifer, "Mission Bay's Sewage Woes", en Los Angeies Times, 15 de junio de 1987. 
